\title{
Infinite decreasing chains in the Mitchell order
}

\author{
Omer Ben-Neria ${ }^{1}$. Sandra Müller ${ }^{2}$ (1)
}

Received: 1 October 2019 / Accepted: 22 January 2021 / Published online: 4 March 2021

(C) The Author(s) 2021

\begin{abstract}
It is known that the behavior of the Mitchell order substantially changes at the level of rank-to-rank extenders, as it ceases to be well-founded. While the possible partial order structure of the Mitchell order below rank-to-rank extenders is considered to be well understood, little is known about the structure in the ill-founded case. The purpose of the paper is to make a first step in understanding this case, by studying the extent to which the Mitchell order can be ill-founded. Our main results are (i) in the presence of a rank-to-rank extender there is a transitive Mitchell order decreasing sequence of extenders of any countable length, and (ii) there is no such sequence of length $\omega_{1}$.
\end{abstract}

Keywords Mitchell order · Rank-to-rank extender · Infinite decreasing chain

Mathematics Subject Classification 03E55 (Large cardinals) · 03E20 (Other classical set theory)

\section{Introduction}

Extenders are combinatorial objects which play a fundamental role in capturing the strength of various large cardinal axioms, and specifically in capturing the strength of elementary embeddings. Given an elementary embedding $j: V \rightarrow M$ with critical point $\kappa$, and an ordinal $\lambda>\kappa$, the $(\kappa, \lambda)$-extender $E$ derived from $j$ is a system of ultrafilters $E=\left(E_{a} \mid a \in[\lambda]^{<\omega}\right)$, where each $E_{a}$ is given by

Sandra Müller

mueller.sandra@univie.ac.at

Omer Ben-Neria

omer.bn@mail.huji.ac.il

1 Omer Ben-Neria, Einstein Institute of Mathematics, The Hebrew University of Jerusalem, Jerusalem 91904, Israel

2 Institut für Mathematik, Universität Wien, Kolingasse 14-16, 1090 Wien, Austria 


$$
X \in E_{a} \text { iff } X \subseteq[\xi]^{|a|} \wedge a \in j(X),
$$

where $\xi \geq \kappa$ is the least ordinal such that $\lambda \leq j(\xi)$. For $a \in[\lambda]^{<\omega}, \mu_{a}$ denotes the least $\mu$ such that $a \subseteq j(\mu)$. Suppose that $a \subseteq b$ are elements of $[\lambda]^{<\omega}$, where $|b|=n$ and $I \subseteq n$ is the set of indices (given by the canonical order on $\lambda$ ) for which $b\lceil I=a$. We note that the map $\pi_{b, a}:\left[\mu_{b}\right]^{|b|} \rightarrow\left[\mu_{a}\right]^{|a|}$ given by $\pi_{b, a}(x)=x \uparrow I$ satifies that for every $X \in E_{a}, \pi_{b, a}^{-1}(X) \in E_{b}$. Each ultrafilter $E_{a}$ is $\kappa$-complete, and the system of ultrafilters naturally gives rise to a canonical system of ultrapowers $\operatorname{Ult}\left(V, E_{a}\right)$. The manner we derived each $E_{a}$ from $j$ implies that $\operatorname{Ult}\left(V, E_{a}\right)$ is isomorphic to the subclass $X_{a}$ of $M$ given by $X_{a}=\left\{j(f)(a) \mid f:\left[\mu_{a}\right]^{|a|} \rightarrow V\right\}$. The functions $\pi_{b, a}$ allow us to form a natural direct limit of the system of ultrapowers, denoted by $\operatorname{Ult}(V, E)$, which is isomorphic to the subclass $X_{E}=\bigcup_{a \in[\lambda]<\omega} X_{a}$ of $M$. We denote the transitive collapse of $\operatorname{Ult}(V, E)$ (which is the same as the transitive collapse of $X_{E}$ ) and the resulting ultrapower embedding by $i_{E}: V \rightarrow M_{E}$. It follows from the description of $X_{E}$ that if the original embedding $j: V \rightarrow M$ is $\lambda$-strong (i.e., $V_{\lambda} \subseteq M$ ) then $V_{\lambda} \subseteq M_{E}$. In that sense, we see that the ultrapower of $V$ by the extender $E$ captures the strength of $j: V \rightarrow M$ up to $\lambda$. The properties of an extender $E=\left(E_{a}, \pi_{b, a} \mid a \subseteq b \in[\lambda]^{<\omega}\right)$ as a system of ultrafilters with suitable connecting maps can be formulated directly, without the need of an ambient embedding $j: V \rightarrow M$. We refer the reader to [9, Section 26] for an extensive introduction to the theory of extenders.

A fundamental notion in the study of extenders is the one of the Mitchell order.

Definition 1.1 Let $E, E^{\prime}$ be two extenders. We write $E \triangleleft E^{\prime}$ if $E$ is represented in the (well-founded) ultrapower of $V$ by $E^{\prime}$.

The Mitchell order $\triangleleft$ was introduced by Mitchell in [10] to construct canonical inner models with many measurable cardinals. The Mitchell order, which was initially introduced as an ordering on normal measures, has been extended to extenders and plays a significant role in inner model theory. As a prominent notion in the theory of large cardinals, the study of the Mitchell order and its structure has expanded in recent decades. The behaviour of the Mitchell order on extenders depends on the type of extenders in consideration and naturally becomes more complicated when restricted to stronger types of extenders. A fundamental dividing line in the behaviour of the Mitchell order is its well-foundedness: Mitchell [11] has shown that $\triangleleft$ is well-founded when restricted to normal measures. The question of the well-foundedness of $\triangleleft$ was further studied by Steel [13], and Neeman [12], who showed that it fails exactly at the level of rank-to-rank extenders.

Definition 1.2 Let $E$ be an extender. We say $E$ is a rank-to-rank extender iff assuming $\lambda>\operatorname{crit}(E)$ is least such that $i_{E}(\lambda)=\lambda$, then $V_{\lambda} \subseteq M_{E}$.

Due to their similarity with embeddings $j: V \rightarrow M$ with $V_{\lambda+2} \subseteq M$, which have been shown by Kunen to be inconsistent with ZFC, the large cardinal strength witnessed by rank-to-rank extenders is considered to be located near the top of the large 
cardinal hierarchy. More precisely, rank-to-rank extenders naturally arise from the large cardinal axiom I2.

The known dividing line of well-foundedness naturally breaks the question of the general possible behaviour into two (i) which well-founded partial orderings can be isomorphic to the Mitchell order on measures/extenders below the rank-to-rank level? and (ii) which ill-founded partial orderings can be isomorphic to the Mitchell order on a set of rank-to-rank extenders? ${ }^{1}$

Concerning question (i), the possible structure of the Mitchell order on normal measures has been extensively studied in [1-5,11,14]. It has been shown in [2] that it is consistent for every well-founded partial ordering to be isomorphic to the restriction of $\triangleleft$ to the set of normal measures on some measurable cardinal $\kappa$ (the exact consistency strength of this property has not been discovered).

In this work, we make a first step towards expanding the study of the Mitchell order in the ill-founded case, and address question (ii). Specifically, we focus on the extent to which the well-foundedness of the Mitchell order fails on rank-to-rank extenders, by considering possible ordertypes of infinite decreasing chains in $\triangleleft$. The main results of this paper are the following two theorems.

Theorem 1.3 Assume there exists a rank-to-rank extender E. Then for every countable ordinal $\gamma$ there is a sequence of rank-to-rank extenders of length $\gamma,\left(E_{\alpha} \mid \alpha<\gamma\right)$, on which the Mitchell order is transitive and strictly decreasing.

Theorem 1.4 There is no $\omega_{1}$-sequence of extenders which is strictly decreasing and transitive in the Mitchell order.

Our presentation of the proof of Theorem 1.4 goes through a proof of a weak version of Steel's conjecture, which addresses transitive $\omega$-sequences of extenders. ${ }^{2}$ This presentation replaces a previous ad-hoc proof. The authors would like to thank Grigor Sargsyan for pointing out the connection with Steel's conjecture, which led to the current concise proof of Theorem 1.4. The (full) conjecture was recently proved by Goldberg ( [7]) building on his remarkable study of the internal relation.

\section{Basic definitions and observations}

For notational clarity it will be sometimes convenient for us to use the notation $\overline{\mathrm{Ult}}(V, E)$ for $M_{E}$, the transitive collapse of $\operatorname{Ult}(V, E)$. For an extender $E$ in a transitive class $N$, write $M_{E}^{N}$ for the transitive collapse of $\operatorname{Ult}(N, E)$ and $i_{E}^{N}: M \rightarrow M_{E}^{N}$ for the corresponding ultrapower embedding. For a rank-to-rank extender $E$ we write $\lambda^{E}$ for the least $\lambda>\operatorname{crit}(E)$ such that $i_{E}(\lambda)=\lambda$. Moreover, we write $\kappa_{0}^{E}=\operatorname{crit}(E)$ and $\kappa_{n+1}^{E}=i_{E}\left(\kappa_{n}^{E}\right)$ for $n<\omega$, and call $\left(\kappa_{n}^{E} \mid n<\omega\right)$ the critical sequence of $E$. For any rank-to-rank extender $E, \lambda^{E}=\sup _{n<\omega} \kappa_{n}^{E}=\operatorname{lh}(E)$.

\footnotetext{
1 Beyond the possible (partial) ordering structure of the Mitchell order, the investigation can be further extended to non-transitive relations, as the Mitchell order need not be transitive in general (see [13]). This direction is not developed in this paper.

2 See Sect. 4 for a formulation of Steel's conjecture.
} 
For every $n<\omega$ let $E\left\lceil\kappa_{n}^{E}\right.$ be the cutback of $E$ to the measures $E_{a}, a \in\left[\kappa_{n}^{E}\right]^{<\omega}$. We have that $M_{E}=\bigcup_{n} X_{n}$ where

$$
X_{n}=\left\{i_{E}(f)(a) \mid a \in\left[\kappa_{n}^{E}\right]^{<\omega}, f:\left[\mu_{a}\right]^{|a|} \rightarrow V\right\} .
$$

By taking $f=\mathrm{id}$, it is clear that $\kappa_{n}^{E} \subseteq X_{n}$. Let $N_{n}$ denote the transitive collapse of $X_{n}$ for each $n<\omega$. We have that $N_{n}$ is isomorphic to $M_{E\left\lceil\kappa_{n}^{E}\right.}$. Moreover, for each $n<\omega$, $N_{n+1}$ contains $V_{\kappa_{n+1}^{E}}$ and in particular $i_{E}$ " $\kappa_{n}^{E} \in N_{n+1}$. It follows from a standard argument that $\kappa_{n}^{E} N_{n+1} \subseteq N_{n+1}$. As the critical point of the inverse of the collapse map $N_{n+1} \rightarrow X_{n+1}$ is $>\kappa_{n}^{E}$, this implies $\kappa_{n}^{E} X_{n+1} \subseteq X_{n+1}$.

The following observations will be useful in comparing extenders in different ultrapowers.

Lemma 2.1 Assume that $N=\bigcup_{n<\omega} X_{n}$ is an increasing union of classes $X_{n}, N$ is transitive, and there exists a sequence of cardinals $\left\langle\kappa_{n} \mid n<\omega\right\rangle$, such that ${ }^{\kappa_{n}} X_{n+1} \subseteq$ $X_{n+1}$ for all $n<\omega$. Let $E \in N$ be a rank-to-rank extender of height $\operatorname{lh}(E)=\lambda=$ $\bigcup_{n} \kappa_{n}$.

(1) For every $a \in[\lambda]^{<\omega}$ and $f:\left[\mu_{a}\right]^{|a|} \rightarrow N$ a function in $V$, there exists a function $f_{N} \in N$ such that $\left\{v \in\left[\mu_{a}\right]^{|a|} \mid f(v)=f_{N}(v)\right\} \in E_{a}$.

(2) Let $i_{E}: V \rightarrow M_{E}$ and $i_{E}^{N}: N \rightarrow M_{E}^{N}$ be the ultrapower embeddings by $E$ of $V$ and $N$, respectively. For every set $x \in N, i_{E}(x)=i_{E}^{N}(x)$.

Proof (1) As $N=\bigcup_{n} X_{n}$ is an increasing union, and $E_{a}$ is $\sigma$-complete, there exists some $n$ such that the set $A_{n}=\left\{v \in\left[\mu_{a}\right]^{|a|} \mid f(v) \in X_{n}\right\}$ belongs to $E_{a}$ and $\mu_{a}<\kappa_{n}^{E}$. Since $X_{n+1}$ is closed under $\kappa_{n}^{E}$-sequences, it follows that $f \uparrow A_{n}$ : $\left[\mu_{a}\right]^{|a|} \rightarrow X_{n}$ belongs to $X_{n+1} \in N$. The claim follows.

(2) Let $\pi: \operatorname{Ult}(V, E) \rightarrow M_{E}$ and $\pi^{N}: \operatorname{Ult}(N, E) \rightarrow M_{E}^{N}$ denote the transitive collapse embeddings. We have that in fact for any $[a, f]_{E}$ and $\left[a, f_{N}\right]_{E}$ for $a \in$ $[\lambda]^{<\omega}, f:\left[\mu_{a}\right]^{|a|} \rightarrow x$ in $V$ and $f_{N}:\left[\mu_{a}\right]^{|a|} \rightarrow x$ in $N$ with $\left\{v \in\left[\mu_{a}\right]^{|a|} \mid\right.$ $\left.f(\nu)=f_{N}(\nu)\right\} \in E_{a}$,

$$
\pi\left([a, f]_{E}\right)=\pi^{M}\left(\left[a, f_{N}\right]_{E}\right) .
$$

This follows from a straightforward induction on the rank of $[a, f]_{E}$ using that for any $[b, g]_{E} \in[a, f]_{E}, g$ takes values in $N$, as $N$ is transitive and we may assume that $g \in N$ by the first part of the lemma. Now this implies $i_{E}(x)=i_{E}^{N}(x)$.

Lemma 2.2 Suppose $E_{2}, E_{1}, E_{0}$ are three rank-to-rank extenders of the same length $\lambda=\lambda^{E_{i}}, i=0,1,2$, such that $E_{2}$ is Mitchell order below $E_{1}$, and both $E_{2}, E_{1}$ are Mitchell order below $E_{0}$. Then $M_{E_{0}}$ also witnesses that $E_{2}$ is Mitchell order below $E_{1}$.

Proof The fact $E_{2} \triangleleft E_{1}$ means that $E_{2}$ is represented in the $V$-ultrapower by $E_{1}$, by a function $f$ and a generator $a$ of $E_{1}$. Take $k<\omega$ such that $a \in\left[\kappa_{k+1}^{E_{1}}\right]^{<\omega}$. 
The reason it is not immediate that the relation $E_{2} \triangleleft E_{1}$ also holds in $M_{E_{0}}$ is that the function $f$ need not belong to $M_{E_{0}}$. However, we can argue that $M_{E_{0}}$ does see some witnessing function $f^{*}$ by using approximations. Assume without loss of generality that $f(v)$ is an extender for every $v \in \operatorname{dom}(f)$. Indeed, notice that for every $n<\omega$, the function $f_{n}$ with $\operatorname{dom}\left(f_{n}\right)=\operatorname{dom}(f)$ such that for every $v, f_{n}(v)$ is the restriction of the extender $f(v)$ to length $\kappa_{n}^{E_{1}}$. Clearly, $\left[a, f_{n}\right]_{E_{1}}^{V}$ represents the cut back of $E_{2}$ to length $\kappa_{n+1}^{E_{1}}$. Moreover, $f_{n}$ belongs to $M_{E_{0}}$ for every $n<\omega$ since $V_{\lambda} \subseteq M_{E_{0}}$.

Let $\mathcal{E}(\eta)$ for some ordinal $\eta$ denote the set of all $(\kappa, \eta)$-extenders. Now, working in $M_{E_{0}}$ and utilizing the fact that both $E_{1}, E_{2}$ belong to the model, we consider the tree $T$ of all pairs $(\tau, n)$ such that $\tau:\left[\kappa_{k}^{E_{1}}\right]^{|a|} \rightarrow \mathcal{E}\left(\kappa_{n}^{E_{1}}\right)$ satisfies that $[a, \tau]_{E_{1}}^{M_{E_{0}}}$ represents the restriction of $E_{2}$ to length $\kappa_{n+1}^{E_{1}}$. The tree order $<_{T}$ is given by $(\tau, n)<_{T}\left(\tau^{\prime}, n^{\prime}\right)$ if $n<n^{\prime}$ and $\tau^{\prime}(\nu)$ extends $\tau(\nu)$ for all $\nu \in \operatorname{dom}(\tau)$. It is clear that a cofinal branch in $T$ translates to a function $F$ for which $[a, F]_{E_{1}}$ represents $E_{2}$, and vice versa. Therefore $f \in V$ witnesses that $T$ has a cofinal branch in $V$, and thus, by absoluteness of well-foundedness, it must also have one in $M_{E_{0}}$.

Steel gives in [13] a folklore example that for rank-to-rank extenders the Mitchell order need not be well-founded. We recall it here because some of the ideas will be used later.

Proposition 2.3 (Folklore) Let E be a rank-to-rank extender. Then there is a strictly decreasing sequence of length $\omega$ in the Mitchell order on which $\triangleleft$ is transitive.

Proof Consider the following sequence of rank-to-rank extenders $\left(E_{n}: n<\omega\right)$. Let $E_{0}=E$ and $E_{n+1}=i_{E_{n}}\left(E_{n}\right)$, where $i_{E_{n}}: V \rightarrow M_{E_{n}}$ is the canonical embedding associated to $E_{n}$. Then it is straightforward to check that every $E_{n}$ is a $V$-extender and $E_{n+1} \triangleleft E_{n}$ for all $n<\omega$.

Claim 1 The Mitchell order is transitive on $\left(E_{n}: n<\omega\right)$.

Proof Let $n<\omega$. We show that $E_{n+2} \triangleleft E_{n}$, the rest follows analogously. By construction $E_{n+2} \in M_{E_{n+1}}$ and $E_{n+1} \in M_{E_{n}}$, we argue that $E_{n+2} \in M_{E_{n}}$. Argue that $i_{E_{n+1}}\left(E_{n+1}\right)=i_{E_{n+1}}^{M_{E_{n}}}\left(E_{n+1}\right)$, where $i_{E_{n+1}}^{M_{E_{n}}}: M_{E_{n}} \rightarrow M_{E_{n+1}}^{M_{E_{n}}}$. By Lemma 2.1, applied to $E=E_{n+1}$ and $M_{E_{n}}$, we see that $E_{n+2}=i_{E_{n+1}}\left(E_{n+1}\right)=i_{E_{n+1}}^{M_{E_{n}}}\left(E_{n+1}\right)$, and hence $E_{n+2} \in M_{E_{n}}$.

\section{Countable decreasing sequences in the Mitchell order}

In this section we prove Theorem 1.3 and show that there can be strictly decreasing transitive sequences in the Mitchell order of any countable length.

Before we turn to the proof, we would like to emphasize why we cannot simply extend the construction in Proposition 2.3 to obtain decreasing sequences of extenders in the Mitchell order of arbitrary (countable) length. Let $E$ be a rank-to-rank extender and let $\left(E_{n}: n<\omega\right)$ be the corresponding sequence of extenders constructed in the 
proof of Proposition 2.3. The critical points of the extenders $E_{n}$ are strictly increasing with supremum $\lambda^{E}$. A natural next extender to consider is the image of $E$ under the direct limit embedding obtained by successively applying the $E_{n}$ 's (internally, i.e., $E_{1}$ is applied to $M_{E_{0}}$ and so forth). This, however, does not work outright as this direct limit embedding moves $\kappa$, the critical point of $E$, to $\lambda$. Instead, we will construct a sequence of extenders $\left(E_{n}^{\prime}: n<\omega\right)$ such that the direct limit embedding $j$ obtained by successively applying the $E_{n}^{\prime}$ 's (again, internally) has critical point strictly above $\kappa$. Now $j(E)$ is a rank-to-rank extender with critical point $\kappa$ and $\lambda^{j(E)}=\lambda$. Moreover, $j(E) \triangleleft E_{n}^{\prime}$ for all $n<\omega$, so if we let $E_{\omega}^{\prime}=j(E),\left(E_{n}^{\prime}: n \leq \omega\right)$ is a decreasing sequence of length $\omega+1$ in the Mitchell order. The proof of Theorem 1.3 elaborates on this idea.

Proof of Theorem 1.3 Let $E$ be a rank-to-rank extender with critical sequence $\left(\kappa_{n} \mid\right.$ $n<\omega)$ and $\lambda^{E}=\lambda$. In what follows, all extenders will have the same length $\lambda$. We start by introducing some notation for sequences of extenders as constructed in the proof of Proposition 2.3. For a rank-to-rank extender $F$, write $S^{1}(F)=i_{F}(F)$ and $S^{n+1}(F)=i_{S^{n}(F)}\left(S^{n}(F)\right)$. Now, the decreasing sequences in the Mitchell order we construct will be of the following form.

Definition 3.1 Let $\vec{E}=\left(E_{\alpha} \mid \alpha<\gamma\right)$ be a sequence of rank-to-rank extenders. Then we say that $\vec{E}$ is guided by an internal iteration iff there are well-founded models $\left(M_{\alpha} \mid \alpha \leq \gamma\right),\left(M_{\alpha}^{*} \mid \alpha \leq \gamma\right)$ with $M_{0}=M_{0}^{*}=V$ and elementary embeddings $j_{\alpha, \beta}^{*}: M_{\alpha}^{*} \rightarrow M_{\beta}^{*}$ for all $\alpha<\beta \leq \gamma$ such that

(1) $E_{\alpha+1}=S^{n}\left(E_{\alpha}\right)$ for some $n \geq 1$ and all $\alpha+1<\gamma$,

(2) $M_{v}=M_{v}^{*}$ for all limit ordinals $v \leq \gamma$ is given as the direct limit of the directed system $\left\langle M_{\alpha}^{*}, j_{\alpha, \beta}^{*} \mid \alpha \leq \beta<v\right\rangle$.

(3) $M_{\alpha+1}=M_{E_{\alpha}}$ and $M_{\alpha+1}^{*}$ is the transitive collapse of $\operatorname{Ult}\left(M_{\alpha}^{*}, E_{\alpha}\right)$. For notational convenience (see the diagram below) we will use $M_{\alpha+1}^{N}$ to denote the transitive collapse of $\operatorname{Ult}\left(N, E_{\alpha}\right)$, therefore $M_{\alpha+1}^{*}=M_{\alpha+1}^{M_{\alpha}^{*}}$.

(4) for all limit ordinals $v<\gamma, E_{v}=j_{0, v}^{*}\left(E_{0}\right)$ and $\operatorname{Ult}\left(V, E_{v}\right)$ is well-founded, and

(5) the following diagram commutes and all maps in the diagram are given by internal ultrapowers.

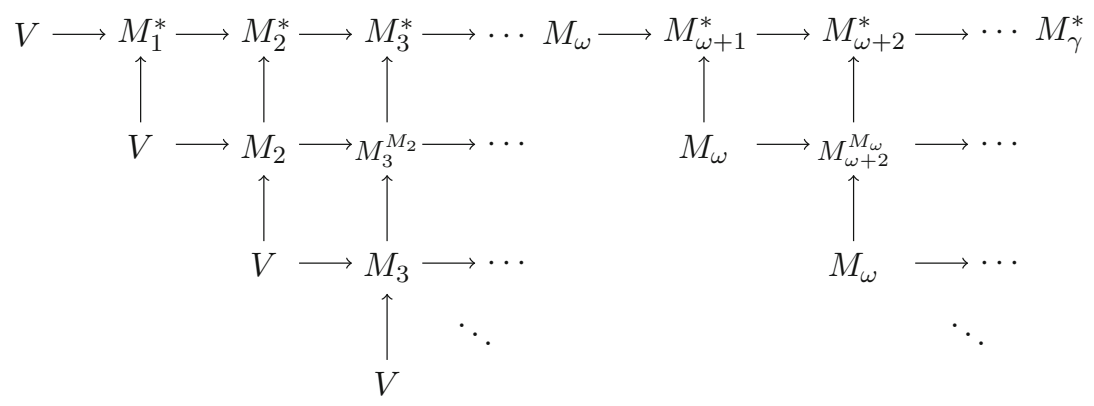

The term guided by an internal iteration refers to the fact that the iteration $\left\langle M_{\alpha}^{*}, j_{\alpha, \beta}^{*} \mid \alpha \leq \beta<\gamma\right\rangle$ is internal, as shown by the next claim. 
Claim 1 For each $\alpha<\gamma, E_{\alpha} \in M_{\alpha}^{*}$.

Proof The claim is immediate when $\alpha$ is a limit ordinal or $\alpha=0$. Let $\alpha=\beta+1$ be a successor ordinal and assume inductively that $E_{\beta} \in M_{\beta}^{*}$.

Suppose first that $E_{\alpha}=S^{n}\left(E_{\beta}\right)$ for $n=1$. Then $E_{\alpha}=i_{E_{\beta}}\left(E_{\beta}\right)$, where $i_{E_{\beta}}: V \rightarrow M_{E_{\beta}}$ is the $V$-ultrapower embedding given by $E_{\beta}$. Recall that $M_{\alpha}^{*}=M_{E_{\beta}}^{M_{\beta}^{*}}$. By applying Lemma 2.1 to $M=M_{\beta}^{*}$ and $E=E_{\beta}$, we conclude that $E_{\alpha}=i_{E_{\beta}}^{M_{\beta}^{*}}\left(E_{\beta}\right) \in$ $M_{E_{\beta}}^{M_{\beta}^{*}}=M_{\alpha}^{*}$. We now know that $S^{1}\left(E_{\beta}\right) \in M_{\alpha}^{*}$, and so for $n>1$, we can further compute $\left(S^{n-1}\right)^{M_{\alpha}^{*}}\left(S^{1}\left(E_{\beta}\right)\right)$ inside $M_{\alpha}^{*}$, which by applying Lemma $2.1(n-1)$ consecutive times, results in

$$
\left(S^{n-1}\right)^{M_{\alpha}^{*}}\left(S^{1}\left(E_{\beta}\right)\right)=S^{n}\left(E_{\beta}\right)=E_{\alpha} .
$$

Before we prove the existence of sequences of extenders guided by internal iterations, we show an abstract claim which will allow us to extend any such sequence by one further element. This is shown as in the proof of Theorem 2.2 in [13] (where this particular argument is attributed to Martin). We sketch the proof here for the reader's convenience.

Claim 2 Let $N \cong \operatorname{Ult}\left(V, E^{\prime}\right)$ for some rank-to-rank extender $E^{\prime}$ of length $\lambda$, and $E \triangleleft F$ rank-to-rank extenders of length $\lambda$ with $E, F \in N$. Then the following diagram commutes and all maps are given by internal ultrapowers.

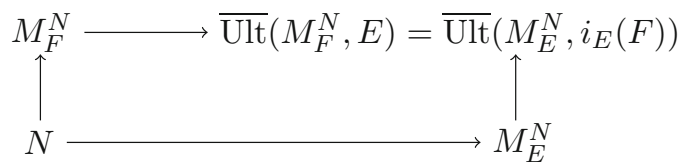

Proof First, we note that $\overline{\operatorname{Ult}}\left(M_{E}^{N}, i_{E}(F)\right)=i_{E}\left(M_{F}^{N}\right)$, where we consider $M_{F}^{N}$ as a class of $N$. Second, we assume $E \triangleleft F$ in $V$, which by Lemma 2.2 implies that $E \triangleleft F$ in $N$ as well, i.e., $E \in M_{F}^{N}$. We can therefore form the internal ultrapower of $M_{F}^{N}$ by $E$. Let $i_{E}^{M_{F}^{N}}: M_{F}^{N} \rightarrow \overline{\operatorname{Ult}}\left(M_{F}^{N}, E\right)$ be the resulting ultrapower embedding. Finally, we have that every $x \in i_{E}\left(M_{F}^{N}\right)$ can be identified with equivalence classes $[a, f]_{E}$, where $a \in[\lambda]^{<\omega}$ and $f:\left[\mu_{a}\right]^{|a|} \rightarrow M_{F}^{N}$, and by Lemma 2.1, $[a, f]_{E}=[a, g]_{E}$ for some function $g \in M_{F}^{N}$. It follows that $i_{E}\left(M_{F}^{N}\right)$ identifies with the internal ultrapower $\operatorname{Ult}\left(M_{F}^{N}, E\right)$, and $i_{E} \uparrow M_{F}^{N}$ with $i_{E}^{M_{F}^{N}}$.

Next, we argue that sequences as in Definition 3.1 are in fact as desired.

Claim 3 If a sequence $\vec{E}=\left(E_{\alpha} \mid \alpha<\gamma\right)$ of extenders is guided by an internal iteration, the embeddings in the diagram witness that $E_{\beta} \triangleleft E_{\alpha}$ for all $\alpha<\beta<\gamma$. In particular, $\vec{E}$ is a decreasing transitive sequence in $\triangleleft$. 
Proof Given a finite ordinal $n<\omega$ and a countable ordinal $\alpha>n$, Claim 1 implies that $E_{\alpha} \triangleleft E_{n}$. This is since all maps in the diagram witnessing that $\vec{E}$ is guided by an internal iteration, are internal ultrapowers, and therefore there are internal iterations from $M_{n+1}$ (the ultrapower of $V$ by $E_{n}$ ) to $M_{n+1}^{*}$ (i.e., vertical maps), and from $M_{n+1}^{*}$ to $M_{\alpha}^{*}$, from which $E_{\alpha}$ is taken.

More generally, given countable ordinals $\beta<\alpha$, we see that $E_{\alpha} \triangleleft E_{\beta}$ by moving first to the last limit ordinal $\eta \leq \beta$ (i.e., $\beta=\eta+n$ for some $n<\omega$ ), and apply the proof of Claim 2 to show that the following diagram commutes and its top row is given by an internal iteration of $M_{E_{\eta+n}}=\overline{\mathrm{Ult}}\left(V, E_{\eta+n}\right)$.

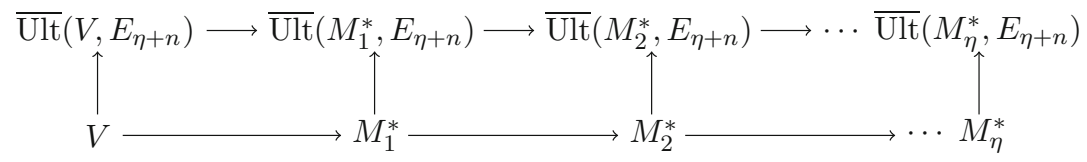

The extenders used to obtain the bottom row of the diagram are $E_{0}, E_{1}$, $E_{2}, \ldots$, while the extenders used to obtain the top row of the diagram are $i_{E_{\eta+n}}\left(E_{0}\right), i_{E_{\eta+n}}^{M_{1}^{*}}\left(E_{1}\right), i_{E_{\eta+n}}^{M_{2}^{*}}\left(E_{2}\right), \ldots$

Finally, we get from $M_{\eta}^{*}$ to $M_{\eta+n}^{*}$ using the same argument described above, for $n<\alpha$. This suffices to show that $E_{\alpha} \triangleleft E_{\eta+n}=E_{\beta}$.

We now turn to proving the following claim, which immediately yields the theorem.

Claim 4 Assume there is a rank-to-rank extendere $E$. For every countable ordinal $\gamma<\omega_{1}$ and an ordinal $\kappa<\lambda=\lambda^{E}$, there is a sequence of rank-to-rank extenders $\vec{E}=\left(E_{\alpha} \mid \alpha \leq \gamma\right)$ which is guided by an internal iteration, such that the induced embedding $j_{0, \gamma}^{*}: V \rightarrow M_{\gamma}^{*}$ satisfies $\kappa<\operatorname{crit}\left(j_{0, \gamma}^{*}\right)<\lambda$ and $j_{0, \gamma}^{*}(\lambda)=\lambda$.

Proof We prove the claim by induction on $\gamma<\omega_{1}$. There is nothing to show for $\gamma=0$. Suppose that $\gamma=1$. Let $\kappa<\lambda$ be arbitrary and fix some $n<\omega$ such that $\kappa<\kappa_{n}^{E}$. Then $E_{0}=S^{n+1}(E)$ and $E_{1}=S^{1}\left(E_{0}\right)$ giving rise to $j_{0,1}^{*}: V \rightarrow M_{E_{0}}$ are as desired.

Now, suppose $\gamma=\alpha+1$ and the claim holds for $\alpha$ witnessed by $\left(E_{v} \mid v \leq \alpha\right)$. Let $n$ be such that $\kappa<\kappa_{n}^{E_{\alpha}}$, the $n$-th element of $E_{\alpha}$ 's critical sequence, and $E_{\alpha+1}=S^{n}\left(E_{\alpha}\right)$. Then $\operatorname{crit}\left(E_{\alpha+1}\right) \geq \kappa_{n}^{\alpha}$ and hence, using that inductively $\operatorname{crit}\left(j_{0, \alpha}^{*}\right)>\kappa$, we have $\operatorname{crit}\left(j_{0, \alpha+1}^{*}\right)>\kappa$. Using Claim 2 the extended sequence $\left(E_{v} \mid v \leq \alpha+1\right)$ is as desired.

Finally, suppose $\gamma<\omega_{1}$ is a limit ordinal and fix an increasing sequence $\left\langle\alpha_{n}\right| n<$ $\omega\rangle$, cofinal in $\gamma$, with $\alpha_{0}=0$. We also fix a well-ordering $<_{w}$ of $H_{\lambda}+$ in $V$.

By the inductive hypothesis applied to $\alpha_{1}$, there is a sequence $\vec{E}^{0}$ of rank-to-rank extenders which is guided by an internal iteration and an elementary embedding $j_{0, \alpha_{1}}^{*}: V \rightarrow M_{\alpha_{1}}^{*}$ with critical point $\nu_{0}>\kappa$ and $j_{0, \alpha_{1}}^{*}(\lambda)=\lambda$. We pick $\vec{E}^{0}$ to be the minimal such sequence with respect to $<_{w}$. By elementarity of $j_{0, \alpha_{1}}^{*}$, we can apply the inductive hypothesis again inside $M_{\alpha_{1}}^{*}$ to get a sequence $\vec{E}^{1}$ of rank-torank extenders which is guided by an internal iteration and an elementary embedding 
$j_{\alpha_{1}, \alpha_{2}}^{*}: M_{\alpha_{1}}^{*} \rightarrow M_{\alpha_{2}}^{*}$ with critical point $\nu_{1}>j_{0, \alpha_{1}}^{*}\left(\nu_{0}\right)$ and $j_{\alpha_{1}, \alpha_{2}}^{*}(\lambda)=\lambda$. We take $\vec{E}^{1}$ to be the minimal such sequence in $M_{\alpha_{1}}^{*}$, with respect to $j_{0, \alpha_{1}}^{*}\left(<_{w}\right)$. Repeating this procedure yields a sequence $\left(\left(\vec{E}^{n}, j_{\alpha_{n}, \alpha_{n+1}}^{*}\right) \mid n<\omega\right)$ of sequences of extenders together with elementary embeddings.

Let $\left(E_{\alpha} \mid \alpha<\gamma\right)$ be the concatenation of the sequences $\vec{E}^{n}, n<\omega$. The choice of $\vec{E}^{n+1}$ to be minimal in $M_{\alpha_{n}}^{*}$ with respect to the well ordering $j_{0, \alpha_{n}}^{*}\left(<_{w}\right)$ guarantees that the sequence $\left\langle\vec{E}^{m} \mid m>n\right\rangle$ belongs to $M_{\alpha_{n}}^{*}$, and thus also the tail of the iteration $\left\langle M_{\alpha}^{*}, j_{\alpha, \beta}^{*} \mid \alpha_{n}<\alpha \leq \beta<\gamma\right\rangle$. Note that $\operatorname{crit}\left(j_{0, \gamma}^{*}\right)>\kappa \operatorname{since} \operatorname{crit}\left(j_{\alpha_{n}, \alpha_{n+1}}^{*}\right)>\kappa$ for all $n<\omega$. Let $j_{0, \gamma}^{*}: V \rightarrow M_{\gamma}^{*}=M_{\gamma}$ be the direct limit embedding of the system.

The reflecting a minimal counterexample argument used to show that internal iterations by normal ultrafilters are well-founded (see e.g., [8, Theorem 19.7] for normal ultrafilters or [6, Proposition 5.8] for rank-to-rank extenders), can also be used to show that $M_{\gamma}$ is well-founded.

Subclaim $1 j_{0, \gamma}^{*}(\lambda)=\lambda$.

Proof Suppose not. Then there is some $\eta<\lambda$ such that $j(\eta) \geq \lambda$. But for every $\eta<\lambda$, there is by choice of the embeddings $j_{\alpha_{n}, \alpha_{n+1}}^{*}$ some $n<\omega$ such that $v_{n}=$ $\operatorname{crit}\left(j_{\alpha_{n}, \alpha_{n+1}}^{*}\right)>\eta$, i.e. $j_{\alpha_{n}, \gamma}^{*}(\eta)=\eta$.

Moreover, it is clear from the construction that, letting $E_{\gamma}=j_{0, \gamma}^{*}(E)$, the resulting sequence $\left(E_{\alpha} \mid \alpha \leq \gamma\right)$ of rank-to-rank extenders is guided by an internal iteration. The only condition that needs a small argument is the following.

Subclaim $2 \operatorname{Ult}\left(V, E_{\gamma}\right)$ is well-founded.

Proof As $M_{\gamma}$ is well-founded and $E_{\gamma}$ is an extender in $M_{\gamma}, \operatorname{Ult}\left(M_{\gamma}, E_{\gamma}\right)$ is wellfounded. We prove the subclaim by defining an elementary embedding $\pi: \operatorname{Ult}\left(V, E_{\gamma}\right)$ $\rightarrow \operatorname{Ult}\left(M_{\gamma}, E_{\gamma}\right)$ as follows. For $[a, f]_{E_{\gamma}}^{V} \in \operatorname{Ult}\left(V, E_{\gamma}\right)$, let

$$
\pi\left([a, f]_{E_{\gamma}}^{V}\right)=\left[a, j_{0, \gamma}^{*}(f) \circ j_{0, \gamma}^{*}\right]_{E_{\gamma}}^{M_{\gamma}} .
$$

$\pi$ is well-defined since for $a \in[\lambda]^{<\omega}, \mu_{a}<\lambda$. Therefore, $j_{0, \gamma}^{*}\left\lceil\left[\mu_{a}\right]^{|a|} \in V_{\lambda} \subseteq M_{\gamma}\right.$. In addition, $j_{0, \gamma}^{*}(f) \circ j_{0, \gamma}^{*}=j_{0, \gamma}^{*} \circ f$, so $\pi$ is elementary.

This finishes the proof of Theorem 1.3.

\section{$4 \mathrm{~A}$ bound on the length of decreasing sequences in the Mitchell order}

Steel proved in [13] that in a Mitchell order decreasing sequence of rank-to-rank extenders, the extenders cannot all have the same critical point. He conjectured the following stronger statement.

Conjecture 1 Suppose that $\left(E_{m} \mid m<\omega\right)$ is a sequence of rank-to-rank extenders which is strictly decreasing in $\triangleleft$. Let $\lambda$ be the unique ordinal such that $\lambda=\lambda^{E_{m}}$ for all sufficiently large $m$. Then $\sup _{m<\omega} \operatorname{crit}\left(E_{m}\right)=\lambda$. 
Theorem 4.1 below establishes Steel's Conjecture for the special case that the Mitchell order is transitive on the sequence $\left(E_{m} \mid m<\omega\right)$.

Theorem 4.1 Suppose that $\left(E_{m} \mid m<\omega\right)$ is a sequence of rank-to-rank extenders, which is strictly decreasing and transitive in $\triangleleft$. Let $\lambda$ be the unique ordinal such that $\lambda=\lambda^{E_{m}}$ for all sufficiently large $m$. Then $\sup _{m<\omega} \operatorname{crit}\left(E_{m}\right)=\lambda$.

Proof Suppose otherwise, and let $\gamma_{0}$ be the minimal ordinal for which there exists a $\triangleleft$ decreasing and transitive sequence $\vec{E}=\left(E_{m} \mid m<\omega\right)$ such that $\gamma_{0}=\sup _{m<\omega} \kappa_{0}^{E_{m}}<$ $\lambda^{\vec{E}}=\sup _{m<\omega} \lambda^{E_{m}}$. We assume without loss of generality that $\lambda^{\vec{E}}=\lambda^{E_{m}}$ for all $m<\omega$. Let $n<\omega$ be the integer for which $\kappa_{n}^{E_{0}} \leq \gamma_{0}<\kappa_{n+1}^{E_{0}}$. We move to the ultrapower $M_{E_{0}}$. By our assumption, $E_{m} \in M_{E_{0}}$ for every $m>0$, and by Lemma 2.2, $M_{E_{0}}$ sees that $E_{m}$ is Mitchell order below $E_{k}$ for every $0<k<m<\omega$. Since $M_{E_{0}}$ is not closed under $\omega$-sequences of its elements (in $V)$ the sequence $\left(E_{m} \mid 1 \leq m<\omega\right)$ need not belong to $M_{E_{0}}$. Nevertheless, we may define in $M_{E_{0}}$ the tree $T^{*}$ of all finite sequences of rank-to-rank extenders $\left(E_{m}^{*} \mid m<N\right)$, which are strictly Mitchell order decreasing, transitive, have length $\lambda^{\vec{E}}$, and satisfy $\gamma_{0} \geq \max _{m<N} \kappa_{0}^{E_{m}^{*}}$. The sequence $\left(E_{m} \mid 1 \leq m<\omega\right.$ ) witnesses that $T^{*}$ has a cofinal branch in $V$. So by absoluteness of well-foundedness there is a cofinal branch in $M_{E_{0}}$ as well. We can now reflect this from $M_{E_{0}}$ back to $V$. Using the fact that $\kappa_{n}^{E_{0}} \leq \gamma_{0}<\kappa_{n+1}^{E_{0}}=i_{E_{0}}\left(\kappa_{n}^{E_{0}}\right)$, we conclude that in $V$, there exists some $\gamma_{-1}<\kappa_{n}^{E_{0}} \leq \gamma_{0}$, and a sequence $\vec{E}^{*}=\left(E_{m}^{*} \mid m<\right.$ $\omega)$ of rank-to-rank extenders which is strictly $\triangleleft$-decreasing and transitive such that $\sup _{m<\omega} \kappa_{0}^{E_{m}^{*}} \leq \gamma_{-1}<\lambda \vec{E}^{*}$. This is a contradiction to the minimality of $\gamma_{0}$.

Now we can obtain Theorem 1.4 as a corollary.

Proof of Theorem 1.4 Suppose otherwise. Let $\vec{E}=\left(E_{\alpha} \mid \alpha<\omega_{1}\right)$ be a sequence of extenders which is strictly decreasing and transitive in the Mitchell order. We may assume that all $E_{\alpha}$ are rank-to-rank extenders and that there exists some $\lambda^{\vec{E}}$ such that $\lambda^{E_{\alpha}}=\lambda^{\vec{E}}$ for all $\alpha<\omega_{1}$. In particular, $\operatorname{cf}\left(\lambda^{\vec{E}}\right)=\omega$ and we may choose a cofinal sequence $\left(\rho_{n} \mid n<\omega\right)$ in $\lambda^{\vec{E}}$. By a straightforward pressing down argument, we can find an uncountable set $I \subseteq \omega_{1}$ and some $n^{*}<\omega$ such that $\kappa_{0}^{E_{\alpha}}<\rho_{n^{*}}$ for all $\alpha \in I$. Taking $\left(\alpha_{n} \mid n<\omega\right)$ to be the first $\omega$ many ordinals of $I$, it follows that $\left(E_{\alpha_{n}} \mid n<\omega\right)$ is strictly decreasing and transitive in the Mitchell order, with $\sup _{n<\omega} \kappa_{0}^{E_{\alpha_{n}}} \leq \rho_{n^{*}}<\lambda^{\vec{E}}$. This contradicts Theorem 4.1.

\section{Questions}

After studying the length of the Mitchell order for rank-to-rank extenders, a natural question that arises is about the structure this order can have.

Question 5.1 Suppose there is a rank-to-rank extender. Can the tree order on the infinite binary tree $2^{<\omega}$ be realized by a Mitchell order?

We can even ask the following more general question. 
Question 5.2 Suppose there is a rank-to-rank extender. Can any tree order on $\omega$ be realized by a Mitchell order?

Acknowledgements This research was initiated whilst the second-listed author visited the first-listed author at the Hebrew University of Jerusalem in February 2019. She wishes to thank the Hebrew University of Jerusalem for its hospitality. The first-listed author was partially supported by the Israel Science Foundation Grant 1832/19. The second-listed author gratefully acknowledges funding from L'ORÉAL Austria, in collaboration with the Austrian UNESCO Commission and in cooperation with the Austrian Academy of Sciences - Fellowship Determinacy and Large Cardinals. The authors would like to thank Grigor Sargsyan and the referee for many valuable comments and suggestions, which greatly improved the presentation of the paper.

Funding Open Access funding provided by University of Vienna.

Open Access This article is licensed under a Creative Commons Attribution 4.0 International License, which permits use, sharing, adaptation, distribution and reproduction in any medium or format, as long as you give appropriate credit to the original author(s) and the source, provide a link to the Creative Commons licence, and indicate if changes were made. The images or other third party material in this article are included in the article's Creative Commons licence, unless indicated otherwise in a credit line to the material. If material is not included in the article's Creative Commons licence and your intended use is not permitted by statutory regulation or exceeds the permitted use, you will need to obtain permission directly from the copyright holder. To view a copy of this licence, visit http://creativecommons.org/licenses/by/4.0/.

\section{References}

1. Baldwin, S.: The $\triangleleft$-ordering on normal ultrafilters. J. Symb. Logic 50(4), 936-952 (1985)

2. Ben-Neria, O.: The structure of the Mitchell order-II. Ann. Pure Appl. Logic 166(12), 1407-1432 (2015)

3. Ben-Neria, O.: The structure of the Mitchell order-I. Isr. J. Math. 214(2), 945-982 (2016)

4. Cummings, J.: Possible behaviours for the Mitchell ordering. Ann. Pure Appl. Logic 65(2), 107-123 (1993)

5. Cummings, J.: Possible behaviours for the Mitchell ordering II. J. Symb. Logic 59(4), 1196-1209 (1994). (12)

6. Dimonte, V.: I0 and rank-into-rank axioms. Bollettino dell'Unione Matematica Italiana 11(3), 315-361 (2018)

7. Gabriel, G.: The internal relation. Submitted, see arXiv:1810.04290

8. Jech, T.J.: Set Theory. Springer Monographs in Mathematics. Springer (2003)

9. Kanamori, A.: The Higher Infinite: Large Cardinals in Set Theory from Their Beginnings. Springer Monographs in Mathematics. Springer (2008)

10. Mitchell, W.J.: Sets constructible from sequences of ultrafilters. J. Sym. Logic 39(1), 57-66 (1974)

11. Mitchell, W.J.: Sets constructed from sequences of measures: revisited. J. Sym. Logic 48(3), 600-609 (1983)

12. Neeman, I.: The Mitchell order below rank-to-rank. J. Symb. Logic 69(4), 1143-1162 (2004)

13. Steel, J.R.: The well-foundedness of the Mitchell order. J. Symb. Logic 58(3), 931-940 (1993)

14. Witzany, J.: Any behaviour of the Mitchell ordering of normal measures is possible. Proc. Am. Math. Soc. 124(1), 291-297 (1996)

Publisher's Note Springer Nature remains neutral with regard to jurisdictional claims in published maps and institutional affiliations. 